\title{
Uso de Gráficos Estatísticos por Futuros Professores dos Primeiros Anos na Realização de Trabalhos de Projeto
}

\section{Use of Statistical Graphs by Prospective Primary School Teachers in Carrying Out Project Works}

\author{
José António Fernandes*a; Maria Helena Martinho; ; Gabriela Gonçalves ${ }^{\mathrm{b}}$
}

${ }^{a}$ Universidade do Minho, Portugal.

'Instituto Politécnico do Porto, Portugal

*E-mail: jfernandes@ie.uminho.pt

\begin{abstract}
Resumo
Neste artigo analisam-se os gráficos estatísticos contruídos por estudantes, futuros professores dos primeiros anos escolares, no âmbito da realização de trabalhos de projeto de natureza investigativa. Os gráficos estatísticos são uma componente importante da literacia estatística ao possibilitarem representar e extrair informação que, por sua vez, permitem resumir, generalizar e revelar tendências nos dados representados. Participaram no estudo 56 estudantes do $2 .^{\circ}$ ano do curso de Licenciatura em Educação Básica, de uma universidade do Norte de Portugal, sendo a sua formação matemática à entrada na universidade muito variada. Para a realização dos trabalhos de projeto os estudantes organizaram-se em pequenos grupos, sendo os dados usados na investigação, ou seja, os gráficos estatísticos, obtidos a partir dos relatórios dos trabalhos de projeto elaborados pelos respetivos grupos. Em termos de resultados do estudo, destaca-se que os estudantes recorreram, sobretudo, a gráficos de barras (simples e múltiplos) e gráficos circulares para representarem os dados, maioritariamente relativos a variáveis estatísticas qualitativas. Já as dificuldades dos estudantes na construção dos gráficos não foram comuns a muitos estudantes, salientando-se a união das barras nos gráficos de barras, o uso de gráficos de linhas para representar dados de variáveis qualitativas e a ausência de etiquetas nos gráficos.
\end{abstract}

Palavras-chave: Gráficos Estatísticos. Trabalhos de Projeto. Futuros Professores. Primeiros Anos Escolares.

\begin{abstract}
In this article are analysed the statistical graphs constructed by students, prospective primary school teachers, in the scope of carrying out investigative project works. Statistical graphs are an important component of statistical literacy as they make it possible to represent and extract information that, in turn, allows to summarize, generalize and reveal trends in the data represented. 56 students from the 2 nd year of the Basic Education Degree course, from a university in Northern of Portugal, participated in the study, with a mathematical training at university entering very varied. To carry out the project work, the students were organized in small groups and the data used in the investigation, that is, the statistical graphs, were obtained from the reports of the project works prepared by the respective groups. In terms of the results of the study, it is noteworthy that students used, above all, bar graphs (single and multiple) and pie charts to represent the data, mostly related to qualitative statistical variables. The difficulties of the students in the construction of the graphs were not common to many students, noting the union of the bars in the bar graphs, the use of line graphs to represent data of qualitative variables and the absence of labels in the graphs.
\end{abstract}

Keywords: Statistical Graphs. Project Works. Prospective Teachers. Primary School.

\section{Introdução}

Atualmente, os gráficos estatísticos são, talvez, a componente mais importante da literacia estatística (Arteaga, Batanero, Cañadas \& Contreras, 2011). As pessoas deparamse constantemente com esse tipo de representação nas mais diversas facetas das suas vidas, seja a nível pessoal ou a nível social. A este respeito, Arteaga et al. (2011) consideram as tabelas e os gráficos estatísticos como objetos culturais, pois tais objetos permitem responder à "necessidade atual de que os cidadãos sejam capazes de tratar diversos tipos de informações estatísticas e suas representações com que se deparam nos diferentes meios de comunicação e em distintos contextos da sua vida" (p. 58).

$\mathrm{Na}$ atividade cognitiva envolvendo gráficos estatísticos identificam-se duas etapas principais: a construção e a leitura e interpretação de gráficos (Friel, Curcio \& Bright, 2001). Nestas duas etapas, começa-se por construir o gráfico ou recorrer a um gráfico contruído por outrem, para de seguida se proceder à sua leitura e interpretação. Segundo Rumsey (2002), na literacia estatística, trata-se de compreender a estatística ao nível de consumidor de informação, portanto numa perspetiva de leitura e interpretação, e ao nível de produtor de informação, correspondente a uma perspetiva de investigação científica. Ambas estas atividades estão intrinsecamente ligadas ao desenvolvimento da literacia estatística e interagem entre si, isto é, a competência de construção de gráficos pode contribuir para o desenvolvimento da competência de leitura e interpretação e vice-versa (Diniz \& Fernandes, 2016).

No caso da construção dos gráficos estatísticos, podem ainda distinguir-se dois momentos sequenciais: num primeiro momento é necessário escolher o tipo de gráfico que é adequado para representar os dados e num segundo momento trata-se de construir o tipo de gráfico que foi antes selecionado. A escolha do tipo de gráfico, enquanto momento 
de tomada de decisão, é especialmente relevante no caso de tarefas estatísticas mais abertas, em que o estudante tem de decidir quais os métodos estatísticos adequados ao estudo da situação que enfrenta, ou em trabalhos de projeto de natureza investigativa. Num estudo recente, envolvendo uma tarefa de natureza aberta, Fernandes e Freitas (2019) verificaram que futuros professores dos primeiros anos tiveram dificuldades em selecionar o tipo de gráfico adequado para representar os dados fornecidos e, posteriormente, em construir os gráficos antes escolhidos.

Neste trabalho, estudantes, futuros professores dos primeiros anos, organizados em pequenos grupos, selecionaram tipos de gráficos e construíram esses gráficos no âmbito da realização de trabalhos de projeto de natureza investigativa. Ora, são esses gráficos, selecionados e construídos pelos estudantes, que aqui são analisados tendo em vista avaliar a diversidade e a adequação dos gráficos usados para representar os dados recolhidos nos respetivos projetos. Assim, ao recorrer a dados obtidos nos trabalhos de projeto, o presente estudo assume-se como complementar dos estudos de Fernandes, Batanero e Gea (2019) e de Fernandes e Freitas (2019), cujos dados resultaram da exploração de uma tarefa aberta.

Uma vez introduzido o estudo, nas secções seguintes desenvolve-se e clarifica-se melhor o enquadramento teórico, descreve-se a metodologia adotada no estudo e apresentase a análise e os resultados obtidos, referentes aos gráficos usados pelos estudantes nos trabalhos de projeto. Por fim, na conclusão sintetizam-se os principais resultados obtidos no estudo e extraem-se algumas implicações didáticas.

\section{Enquadramento Teórico}

$\mathrm{Na}$ realização de trabalhos de projeto de natureza investigativa, em que se espera que os intervenientes (neste caso, estudantes) criem conhecimento, tem-se de percorrer várias etapas, desde o estabelecimento do problema a investigar até às conclusões do estudo. No caso da Estatística, Wild e Pfannkuch (1999), referindose ao ciclo investigativo PPDAC, estabeleceram as cinco etapas seguintes: problema $(\mathrm{P})$ - existe um problema que interessa resolver (formulação do problema e hipóteses ou objetivos); plano (P) - plano geral de resolução do problema (apresentação de um plano genérico do estudo, designadamente no que concerne aos dados utilizados e à sua análise); dados (D) - obter dados tendo em vista resolver o problema (descrever detalhadamente os dados usados no estudo); análise (A) - tratar e analisar os dados (referir e aplicar os métodos de organização e tratamento dos dados estatísticos); e conclusões (C) - avaliar os resultados obtidos no estudo (apresentar as respostas obtidas para o problema ou voltar à segunda etapa do ciclo caso não se tenha resolvido o problema). De entre estas etapas, no presente estudo envolvem-se, principalmente, a segunda e quarta etapas, ou seja, as etapas de planificação e análise.

Segundo Batanero, Díaz, Contreras e Arteaga (2011), a realização de trabalhos de projeto é hoje muito valorizada no campo da educação estatística, na medida em que promove a motivação e potencializa os significados da estatística ao salientar os contextos e a sua natureza realista. Adicionalmente, para estes autores, tarefas deste tipo permitem aos estudantes desenvolver conhecimentos estratégicos, que se acrescentam aos conhecimentos técnicos praticados com tarefas mais convencionais. Gonçalves, Fernandes e Gonçalves (2020) constataram que estudantes do ensino superior percecionaram tais vantagens do trabalho de projeto quando aprenderam sobre testes de hipóteses seguindo essa metodologia.

No mesmo sentido, Godino, Arteaga, Estepa e Rivas (2013) preconizam que o ensino da Estatística deve centrarse na exploração de trabalhos de projeto, enquanto estratégia para dar sentido às técnicas e às teorias estatísticas. Estes autores conduziram um estudo com futuros professores dos primeiros anos sobre Probabilidades e Estatística, em que recorreram a uma metodologia de trabalhos de projeto em análise de dados, onde concluíram que os estudantes revelaram dificuldades concetuais e procedimentais, que requerem uma maior atenção do professor nos momentos de exercitação e institucionalização dos conhecimentos.

No caso dos gráficos estatísticos, num trabalho de projeto ou em tarefas abertas, geralmente, os estudantes têm de responder a duas questões sequenciais: 1) selecionar um tipo de gráfico que seja adequado para representar os dados e, seguidamente, 2) construir o gráfico antes escolhido (Arteaga, Díaz-Levicoy \& Batanero, 2017). Repare-se que no caso das tarefas fechadas, geralmente, a primeira questão é omitida, sendo requerido ao estudante que construa um tipo de gráfico especificado.

Na seleção do tipo de gráfico estatístico, o tipo de variável estatística desempenha um papel importante ao condicionar os tipos de gráficos que são adequados para representar os dados. Assim, o tratar-se de dados qualitativos ou quantitativos, discretos ou contínuos, em número pequeno ou elevado e com muita ou pouca repetição tem implicações na escolha do gráfico adequado para representar esses dados. Ora, esta variedade de aspetos que se devem ter em conta na escolha do gráfico mostra o quão difícil pode ser para os estudantes essa seleção (Fernandes, Morais \& Lacaz, 2011). Para além da classificação dos gráficos decorrente do tipo de variável estatística, no presente estudo, classificamos também os gráficos em simples e múltiplos consoante representam uma e duas variáveis estatísticas, respetivamente.

No estudo de Fernandes et al. (2019), em que participaram futuros professores dos primeiros anos, foi pedido aos estudantes que representassem os dados de uma variável qualitativa nominal (bebida preferida pelos 20 alunos de uma turma) através de um gráfico adequado. Nesse estudo, verificou-se que cerca de três em cada quatro estudantes, ou 
mesmo mais, indicaram um gráfico de barras e/ou circular, os quais constituem gráficos adequados para representar os dados. Mesmo assim, 28\% dos estudantes indicaram o histograma, o gráfico de linhas ou o diagrama de extremos e quartis, que são gráficos não adequados para representar dados de uma variável qualitativa nominal.

Já no estudo de Fernandes e Freitas (2019), em que participaram futuros professores dos primeiros anos, foi pedido para indicarem todos os tipos de gráficos adequados para representar os dados de uma variável quantitativa discreta (classificações obtidas num teste Matemática pelos 12 alunos de uma turma, numa escala de 0 a 100) e, de seguida, escolher um desses tipos de gráfico e construí-lo. Dos resultados obtidos, salienta-se que, em média, cada estudante indicou dois gráficos, sendo muitos mais os gráficos não adequados (56) do que os adequados (38).

Relativamente aos tipos de gráficos indicados pelos estudantes, considerados adequados, verificou-se que o histograma ${ }^{1}$ foi referido por mais de metade dos estudantes (70\%), seguindo-se o diagrama de caule-e-folhas com uma frequência muito reduzida $6 \%$ ). Ainda neste caso, constatouse que não foi indicado o diagrama de extremos e quartis, apesar de ser um gráfico adequado para representar a série de dados. Já no caso dos gráficos não adequados, o gráfico de barras foi o mais selecionado (72\%), seguindo-se o gráfico de linhas (14\%), o gráfico circular e o diagrama de dispersão (ambos com 10\%), a tabela de frequências (4\%) e o gráfico de barras agrupadas $(2 \%)$.

Já relativamente aos gráficos construídos pelos estudantes, considerados adequados, verificou-se que quase metade (48\%) construiu um histograma e poucos (4\%) construíram um diagrama de caule-e-folhas, enquanto nos gráficos não adequados se incluem o gráfico de barras $(24 \%)$, o gráfico de linhas $(6 \%)$ e a tabela de frequências $(2 \%)$. Contudo, no caso do histograma, muitos estudantes (38\%) usaram 5, 6 ou 10 classes, quando, para um conjunto de 12 dados, seria adequado usar 3 ou 4 classes (recorrendo à Tabela de Truman L. Kelly (Toledo \& Ovalle, 1985) ou à regra da raiz quadrada da dimensão da amostra).

Observa-se, assim, resultados muito díspares nos dois estudos antes referidos, o que se explica pelo facto de o gráfico de barras ser um tipo de gráfico adequado no primeiro estudo (Fernandes et al., 2019) e não adequado no segundo estudo (Fernandes e Freitas, 2019). Sendo este tipo de gráfico muito praticado na escola, desde os primeiros anos escolares, é mais provável que os estudantes o usem em detrimento de outros tipos de gráfico, tal como também Fernandes et al. (2011) verificaram em alunos do $9 .^{\circ}$ ano.

Por fim, nos estudos de Fernandes et al. (2019) e de Fernandes e Freitas (2019), antes referidos, observou-se que muitos gráficos construídos apresentavam omissões, designadamente, ao não terem título, ao não nomearem os eixos coordenados e ao não incluírem a legenda. A omissão desta informação dificulta a leitura e interpretação dos gráficos, constituindo, portanto, um aspeto crítico da sua construção (Friel et al., 2001).

\section{Metodologia}

Neste estudo analisam-se os gráficos estatísticos usados por estudantes, futuros professores dos primeiros anos, na realização de projetos estatísticos de natureza investigativa.

$\mathrm{O}$ envolvimento dos estudantes em trabalhos de projeto permite-lhes desenvolver uma aprendizagem mais profunda do que no caso da exploração de tarefas fechadas, em que são omitidas muitas etapas do processo investigativo. No caso dos gráficos estatísticos, que aqui são estudados, frequentemente é-lhes solicitada a construção de um gráfico específico, o que implica que os estudantes não terão oportunidade de decidir sobre o tipo de gráfico que é adequado para representar os dados com que se deparam.

Participaram no estudo 57 estudantes do $2 .^{\circ}$ ano do curso de Licenciatura em Educação Básica, de uma universidade do Norte de Portugal. Estes estudantes, futuros professores dos primeiros anos, tinham uma formação muito variada em termos de conhecimentos matemáticos estudados antes da sua entrada na universidade, que ia desde a formação matemática obtida no final do $9 .^{\circ}$ ano (que é o último ano do ensino básico), em cursos profissionais, em cursos humanísticos e em cursos científico-tecnológicos, cursos estes inseridos no ensino secundário.

Os estudantes organizaram-se em 18 pequenos grupos (designados por, com ), sendo 16 grupos com três estudantes, um grupo com quatro estudantes e um grupo com cinco estudantes, para realizarem os trabalhos de projeto, de natureza investigativa, envolvendo Estatística. Estes trabalhos realizaram-se no âmbito da unidade curricular de Probabilidades e Estatística e fizeram parte da avaliação formal da unidade curricular. Os trabalhos de projeto foram desenvolvidos, fundamentalmente, fora das aulas da unidade curricular, embora tenha havido uma aula presencial em que os estudantes puderam trabalhar nos seus projetos e esclarecer dúvidas com o docente da unidade curricular. Os estudantes puderam também discutir e clarificar com o docente aspetos dos seus projetos nas aulas de tutoria da unidade curricular.

Os dados usados no presente estudo foram obtidos através dos relatórios dos trabalhos de projeto realizados pelos grupos de estudantes, num total de 18 relatórios, mais propriamente, usaram-se os gráficos que constavam desses relatórios. $\mathrm{Na}$ construção dos gráficos, à semelhança do que aconteceu nas aulas da unidade curricular de Probabilidades e Estatística, os estudantes puderam recorrer a uma folha de cálculo ou a uma

1 Embora a variável em estudo seja discreta, o facto de os dados serem quase todos distintos requer que a variável seja tratada como contínua para efeitos de análise estatística. 
calculadora gráfica.

Em termos de análise dados, classificaram-se os gráficos estatísticos segundo os tipos de gráficos e segundo o tipo de variável estatística referente aos dados, determinando-se frequências segundo esses atributos e sintetizando-as numa tabela de dupla entrada para os casos dos gráficos simples e dos gráficos múltiplos. Ao longo de todo o processo de análise destacam-se também as dificuldades sentidas pelos estudantes na construção dos gráficos estatísticos, tendo em consideração a adequação do tipo de gráfico escolhido e a sua construção. Por fim, apresentam-se exemplos dos gráficos construídos pelos estudantes com o propósito de exemplificar e melhor compreender os gráficos por eles construídos.

\section{Uso de Gráficos na Análise de Dados de Trabalhos de Projeto}

$\mathrm{Na}$ análise de dados, realizada pelos estudantes dos vários grupos, constata-se que muitos deles usaram gráficos para resumir os dados dos seus projetos. Ora, são esses gráficos, construídos pelos estudantes, que são aqui estudados, tendo em conta as duas seguintes categorias: gráficos simples e gráficos múltiplos.

\subsection{Construção de gráficos simples}

Os gráficos simples permitem representar dados de uma só variável estatística. $\mathrm{Na}$ Tabela 1 apresentam-se os tipos de gráficos simples construídos pelos estudantes, segundo o tipo de variável estatística envolvida.

Tabela 1 - Frequência de tipos de gráficos simples construídas pelos estudantes

\begin{tabular}{|c|c|c|c|c|c|}
\hline \multirow[b]{2}{*}{ Tipo de Gráfico } & \multicolumn{4}{|c|}{ Tipo de Variável Estatística } & \multirow[b]{2}{*}{ Total } \\
\hline & Qualitativa nominal & Qualitativa ordinal & $\begin{array}{c}\text { Quantitativa } \\
\text { discreta }\end{array}$ & $\begin{array}{c}\text { Quantitativa } \\
\text { contínua }\end{array}$ & \\
\hline Barras simples & 41 & 11 & 24 & - & 76 \\
\hline Circular & 55 & 11 & 6 & 3 & 75 \\
\hline Linhas simples & 3 & 2 & - & - & 5 \\
\hline Histograma & - & - & 1 & 16 & 17 \\
\hline Diagrama de caule-e-folhas & - & - & 2 & - & 2 \\
\hline Diagrama de extremos e quartis & - & - & 2 & - & 2 \\
\hline Pictograma & 1 & - & - & - & 1 \\
\hline Total & 100 & 24 & 35 & 19 & 178 \\
\hline
\end{tabular}

De entre os tipos de gráficos, verifica-se que os estudantes recorreram mais frequentemente ao gráfico de barras simples e ao gráfico circular do que aos outros tipos de gráficos. Seguidamente, com uma frequência inferior, recorreram ao histograma e ao gráfico de linhas simples, enquanto o diagrama de caule-e-folhas, o diagrama de extremos e quartis e o pictograma raramente foram usados para representação de dados. A prevalência destes tipos de gráficos pode dever-se ao facto de eles terem sido mais trabalhados nas aulas destes estudantes (Morais \& Fernandes, 2011), seja antes de entrarem na universidade, seja mesmo na universidade.

Assim, conclui-se que os estudantes recorreram a tipos de gráficos mais tradicionais, característicos da Estatística Descritiva, e quase nunca usaram gráficos mais recentemente criados no âmbito da Análise Exploratória de Dados (Tukey, 1977), como sejam o diagrama de caule-efolhas e o diagrama de extremos e quartis. Esta tendência também é referida por Fernandes et al. (2011), quando alunos do $9 .^{\circ}$ recorreram maioritariamente ao gráfico de barras simples, e por Fernandes et al. (2019), quando estudantes, com formação semelhante aos participantes deste estudo, recorreram, mais frequentemente, aos gráficos de barras simples e circular para representar os dados, comparativamente com o histograma, o gráfico de linhas simples e o diagrama de extremos e quartis.

Também o quase não uso do pictograma, que apenas foi usado uma vez, parece um tanto problemático na medida em que este tipo de gráfico é habitualmente usado pelos alunos dos primeiros anos escolares. Acrescenta-se, ainda, que este tipo de gráfico é referido explicitamente logo no $1 .^{\circ}$ ano do programa oficial da disciplina de Matemática do ensino básico (Ministério da Educação e Ciência, 2013).

Em termos do tipo de variável estatística, referente aos dados, constata-se que mais de metade dos gráficos construídos pelos estudantes envolve uma variável estatística do tipo qualitativo nominal, tipo esse que também é largamente maioritário nos gráficos de barras simples e circulares. Com cerca de um quinto do total das frequências surgem as variáveis quantitativas discretas, cujos dados são representados por praticamente todos os tipos de gráficos, com cerca de um oitavo do total das frequências surgem as variáveis qualitativas ordinais, com maior incidência nos gráficos de barras simples e circulares, e, por fim, com cerca de um nono do total das frequências surgem as variáveis quantitativas contínuas, praticamente representadas através de histogramas. No caso das variáveis quantitativas contínuas, três estudantes recorreram a um gráfico circular, como se exemplifica na Figura 1. 
Figura 1 - Gráfico circular construído pelo grupo G2
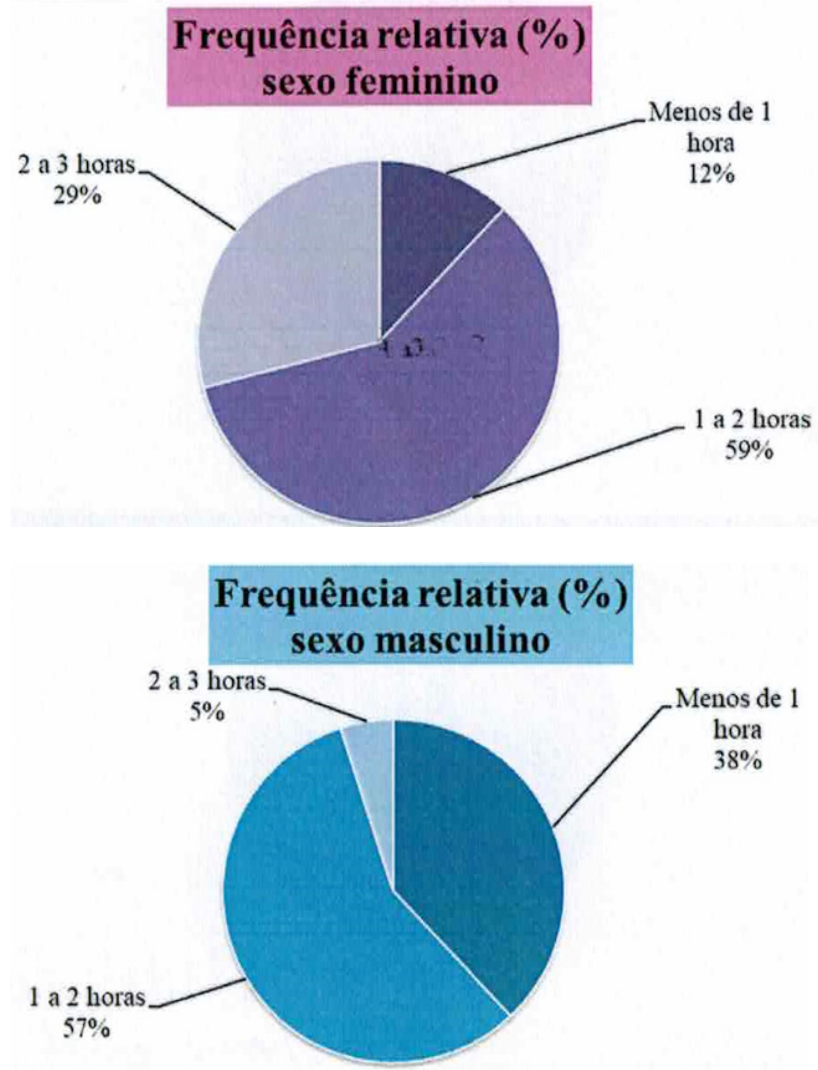

Us do1s gráticos circulares, construidos pelo grupo $\mathrm{G} 2$, e relativos a cada um dos sexos, apresentam as percentagens de alunos do 2. ${ }^{\circ}$ ciclo segundo o tempo de estudo diário. Para tal, os alunos consideraram esse tempo agrupado em três classes: menos de $1 \mathrm{~h}$, entre $1 \mathrm{~h}$ e $2 \mathrm{~h}$ e entre $2 \mathrm{~h}$ e $3 \mathrm{~h}$, embora nem sempre seja claro a classe a que pertencem os valores extremos.

Dificuldades

Seguidamente vamo-nos referir às principais dificuldades e erros revelados pelos estudantes na construção dos gráficos simples. No caso dos gráficos de barras simples, dois grupos uniram as barras dos gráficos em que se representavam variáveis qualitativas nominais (11 gráficos) e variáveis quantitativas discretas (2 gráficos), como se de variáveis contínuas se tratasse. No gráfico da Figura 2 apresenta-se um exemplo de um desses gráficos.

Figura 2 - Gráfico de barras simples construído pelo grupo G3. Disciplina de que menos gosta (masculino)

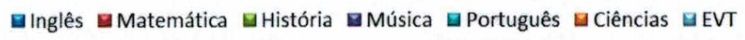

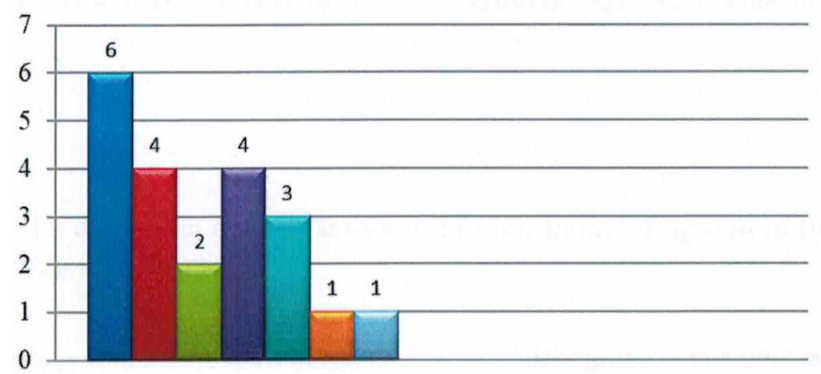

O grupo G3 uniu as barras do gráfico de barras simples que construiu, referente à variável "disciplina de que menos gosta" no universo dos alunos de uma turma do $2{ }^{\circ}$ ciclo de uma escola. Ora, tratando-se de uma variável qualitativa nominal, não se deve unir as barras do gráfico; enquanto que no caso das variáveis quantitativas discretas, em que há pouca repetição de dados, é adequado considerá-las como contínuas para efeitos de análise estatística e, em consequência, unir as barras respetivas.

Três dos gráficos circulares construídos pelos grupos apresentam um número excessivo de setores e/ou setores muito pequenos, o que dificulta a leitura do gráfico. Nestas situações, Silva (2006) desaconselha o recurso a este tipo de gráficos. Já os cinco gráficos de linhas simples representam dados de uma variável qualitativa nominal ou ordinal. Contudo, esse tipo de gráfico é adequado para representar variáveis quantitativas contínuas, usualmente envolvendo uma sequência temporal. Na Figura 3 podemos observar um desses gráficos.

Figura 3 - Gráfico de linhas simples construído pelo grupo G7

\section{Cores dos Animais}

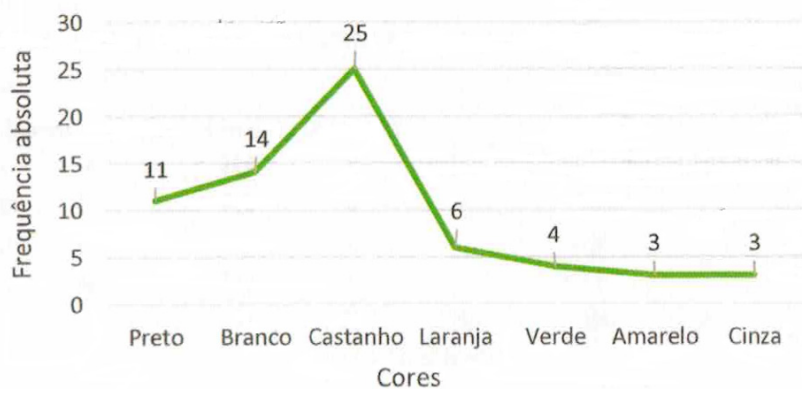

O gráfico construído pelo grupo G7, envolvendo a variável "cores dos animais" dos alunos do 4. ${ }^{\circ}$ ano de uma escola, variável do tipo qualitativa nominal, não é adequado para representar os dados em questão. Essa desadequação está bem patente no facto de não ser possível estabelecer uma relação de ordem (crescente) entre os valores da variável estatística e, em consequência, de se poder obter diferentes linhas para diferentes sequências dos valores da variável.

A adesão a ideias erradas, como seja unir a barras num gráfico de barras ou recorrer a gráficos de linhas para representar variáveis qualitativas, é muito problemática pois, tratando-se de aspetos concetuais dos gráficos estatísticos, o seu enraizamento cognitivo condicionará fortemente as aprendizagens subsequentes dos estudantes (Fernandes, 1990). Além disso, esses erros podem ter consequências negativas no ensino dos seus futuros alunos na medida em que o seu conhecimento e as suas experiências anteriores certamente influenciarão o seu ensino (Brown \& Borko, 1992).

\subsection{Construção de gráficos múltiplos}

Diferentemente dos gráficos simples, que permitem representar dados relativos a única variável, os gráficos múltiplos permitem representar dados relativos a duas variáveis para estabelecer comparações entre subconjuntos do conjunto total de dados ou estabelecer relações entre duas variáveis estatísticas. Segundo Silva (2006), esta segmentação 
dos dados em subconjuntos é definida por uma outra variável que se cruza com a variável referente ao conjunto total de dados. Nos projetos realizados pelos vários grupos verificou- se que, frequentemente, essa segmentação foi estabelecida pela variável "sexo". Na Tabela 2 apresentam-se os tipos de gráficos múltiplos usados pelos grupos nos seus relatórios.

Tabela 2 - Tipos de gráficos múltiplos construídos pelos estudantes

\begin{tabular}{|l|c|c|c|c|c|}
\hline \multirow{2}{*}{ Tipo de Gráfico } & \multicolumn{4}{|c|}{ Tipo de variável estatística } & \\
\cline { 2 - 6 } & Qualitativa nominal & Qualitativa ordinal & Quantitativa discreta & Quantitativa contínua & Total \\
\hline Barras agrupadas & 20 & 10 & 6 & - & 36 \\
\hline Barras empilhadas & 6 & 1 & 1 & - & 8 \\
\hline Linhas múltiplas & 4 & 2 & 6 & - \\
\hline Diagrama de dispersão & - & - & 8 & - \\
\hline \multicolumn{1}{|c|}{ Total } & 30 & 13 & 21 & - \\
\hline
\end{tabular}

No conjunto dos gráficos múltiplos usados pelos grupos, mais de metade são gráficos de barras agrupadas, seguindo-se o número de gráficos de linhas múltiplas e, por fim, o número de gráficos de barras empilhadas e de diagramas de dispersão. Já em termos do tipo de variável estatística envolvida, cerca de metade dos gráficos representam variáveis qualitativas nominais, seguese o número de gráficos envolvendo variáveis quantitativas discretas e, finalmente, o número de gráficos envolvendo variáveis qualitativas ordinais é o menor. A prevalência das variáveis qualitativas nominais também se verificou num estudo, envolvendo futuros professores dos primeiros anos, sobre a construção de tabelas de frequências na realização de trabalhos de projeto (Fernandes, Gonçalves \& Barros, 2021), podendo essa preferência dos estudantes dever-se a uma análise estatística mais simples e às suas próprias experiências nessas situações.

\section{Dificuldades}

Em termos das dificuldades sentidas pelos estudantes verifica-se que, excetuando um gráfico de barras empilhadas e um de linhas múltiplas, em todos os outros tipos de gráficos os estudantes usaram as frequências absolutas, o que não constitui um tipo de frequência adequado para comparar amostras de diferentes dimensões. Para ultrapassarem esta dificuldade, os estudantes poderão ser confrontados com exemplos extremos, ou seja, em que a incidência de um atributo seja muito diferente nas duas amostras.

À semelhança do que se verificou com os gráficos de linhas simples, os estudantes não usaram os gráficos de linhas múltiplas para representar variáveis quantitativas contínuas, incluindo sequências temporais em que normalmente são utilizados. $\mathrm{Na}$ Figura 4 apresenta-se um exemplo deste tipo de gráfico.

Figura 4 - Gráfico de linhas múltiplas construído pelo grupo G6. $\mathrm{O}$ que usas para brincar?

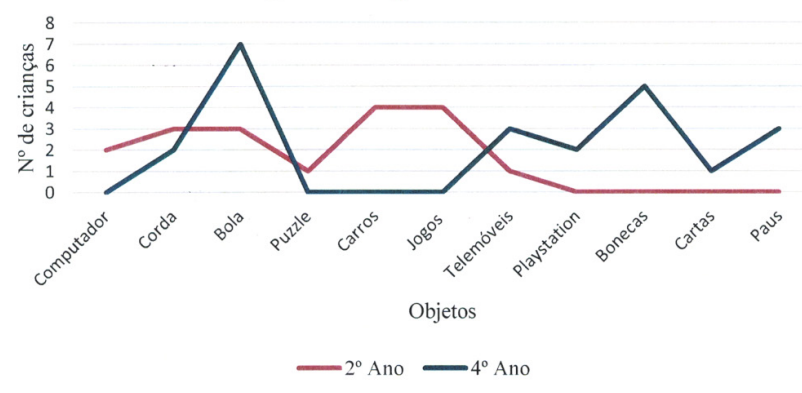

Pela Figura 4 verifica-se que os estudantes do grupo G6 construíram um gráfico de linhas múltiplas para dados de uma variável qualitativa nominal, relativa aos "objetos" que alunos do $2 .^{\circ}$ e $4 .^{\circ}$ ano usam para brincar. Face a este tipo de variável estatística, o gráfico é totalmente desadequado pois obtêm-se diferentes linhas do gráfico para diferentes sequências dos valores da variável que se considere, tal como foi referido antes para os gráficos de linhas simples. Adicionalmente, na construção do gráfico, os estudantes recorreram a frequências absolutas, o que dificulta a comparação entre os dois subgrupos considerados, os alunos do $2 .^{\circ}$ ano e os do $4 .^{\circ}$ ano, que têm um número total de alunos diferente.

Por último, dos oito diagramas de dispersão, envolvendo variáveis quantitativas discretas, em quatro não faz sentido estabelecer qualquer correlação ou regressão linear entre as variáveis implicadas. Na Figura 5 apresenta-se um exemplo de diagrama de dispersão em que é patente essa ausência de relação entre as variáveis.

Figura 5 - Diagrama de dispersão construído pelo grupo G2 Classificação dos testes das disciplinas de que menos gosta

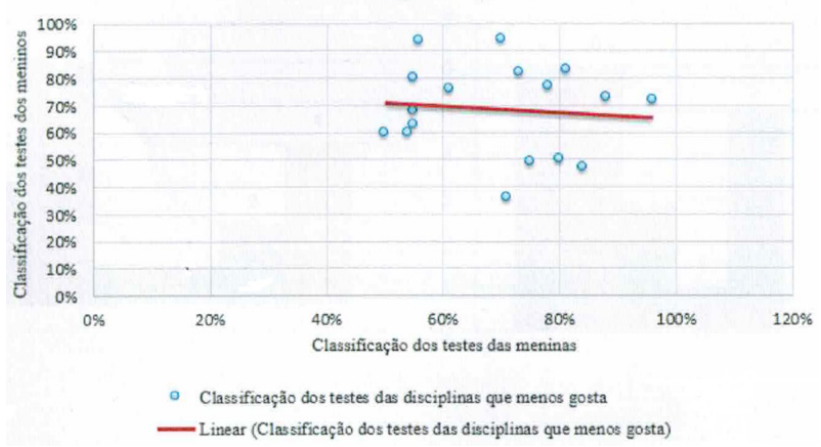

No diagrama de dispersão construído pelo grupo G2 estão em jogo as variáveis "classificação dos testes das meninas" e "classificação dos testes dos meninos", relativas à disciplina que os alunos do $2{ }^{\circ}$ ciclo menos gostam. Nesta situação não se percebe como foram definidos os pontos do diagrama, pois as variáveis em estudo não constituem uma distribuição bidimensional. Em consequência, não sendo possível estabelecer qualquer relação entre os valores da variável, o grupo estabeleceu uma relação arbitrária. Num estudo realizado por Estepa (2007), envolvendo estudantes 
do último ano do ensino secundário, verificou-se que apenas cerca de metade identificaram corretamente a distribuição bidimensional num conjunto de quatro distribuições indicadas.

Finalmente, na totalidade dos gráficos, alguns apresentavam falhas na sua estrutura, especificamente, não tinham título (3 gráficos), não intitulavam o eixo horizontal (63 gráficos), não intitulavam o eixo vertical (51 gráficos) e não especificavam os valores dos setores circulares (1 gráfico).

\section{Conclusão}

Na totalidade dos gráficos construídos pelos estudantes, futuros professores dos primeiros anos, em termos de frequências, salienta-se o gráfico de barras (simples e múltiplos) e o gráfico circular, que globalmente correspondem a cerca de $80 \%$ do número total de gráficos dos relatórios dos trabalhos de projeto.

Já em termos das variáveis estatísticas envolvidas nos gráficos, em termos de frequências, destaca-se claramente o tipo de variável qualitativa nominal, em mais metade dos gráficos, seguindo-se o tipo de variável quantitativa discreta, em cerca de um quarto dos gráficos. Globalmente, verificase que, na maior parte dos casos, os estudantes construíram gráficos compatíveis com o tipo de variável estatística, o que contrasta com os resultados do estudo de Fernandes e Freitas (2019). Neste último estudo estava envolvida uma variável quantitativa discreta (com pouca repetição de dados), o que implica que tanto o gráfico de barras como o gráfico circular não são adequados para representar tais dados, o que é diferente no presente estudo, em que, maioritariamente, os estudantes consideraram variáveis qualitativas nominais.

Apesar da maioria dos estudantes ter selecionado gráficos adequados ao tipo de variável estatística, eles revelaram várias dificuldades na construção desses gráficos, designadamente: ao unir as barras nos gráficos de barras (simples); ao usar no gráfico circular demasiados setores e/ou setores muito pequenos; ao recorrerem a gráficos de linhas (simples e múltiplos) para representar dados de variáveis qualitativas nominais e ordinais; ao recorrerem a digramas de dispersão para representar duas variáveis que não definem uma distribuição bidimensional (Estepa, 2007); ao usar frequências absolutas quando estava em questão a comparação de amostras de diferentes dimensões; e ao não escrever um título e/ou não nomear os seus eixos.

De entre os erros referidos, destacam-se aqueles que estão na origem dos próprios conceitos, como acontece com a união das barras nos gráficos de barras e a construção de gráficos de linhas para variáveis qualitativas. Mesmo não sendo comuns a muitos estudantes, os erros por eles cometidos devem ser enfrentados ao longo da sua formação inicial e contínua, sempre numa perspetiva de explorar o erro como uma oportunidade de aprender (Barros, Fernandes \& Araújo, 2016).

\section{Referências}

Arteaga, P., Batanero, C., Cañadas, G., \& Contreras, J. M. (2011). Las tablas y gráficos estadísticos como objetos culturales. Números, 76, 55-67.

Arteaga, P., Díaz-Levicoy, D., \& Batanero, C. (2017). Investigaciones sobre gráficos estadísticos en Educación Primaria: revisión de la literatura. Revista Digital Matemática Educación e Internet, 18(1).

Barros, P. M., Fernandes, J. A., \& Araújo, C. M. (2016). Perspetivas dos alunos sobre o erro como estratégia de aprendizagem. In M. H. Martinho, R. A. Tomás Ferreira, I. Vale \& H. Guimarães (Eds.), Atas do XXVII Seminário de Investigação em Educação Matemática (pp. 119-131). Porto: Associação de Professores de Matemática.

Batanero, C., Díaz, C., Contreras, J. M.; Arteaga, P. (2011). Enseñanza de la Estadística a través de proyectos. In C. Batanero \& C. Díaz (Ed.), Estadística con Proyectos (pp. 9-46). Granada: Universidad de Granada.

Brown, C., \& Borko, H. (1992). Becoming a mathematics teacher. Em D. Grouws (Ed.), Handbook of research on mathematics teaching and learning (pp. 209-239). New York, NY: Macmillan.

Diniz, L. N., \& Fernandes, J. A. (2016). Interações entre construção e interpretação de gráficos estatísticos em projetos de modelagem matemática com uso de tecnologias de informação e comunicação. VIDYA, 36(2), 457-475.

Estepa,A. (2007). Caracterización del significado de la correlación y regresión en estudiantes de educación secundaria. Zetetiké, 15(28), 119-152.

Fernandes, J. A. (1990). Concepções erradas na aprendizagem de conceitos probabilísticos. Dissertação (Mestrado em Informática no Ensino), Universidade do Minho, Braga, Portugal.

Fernandes, J. A., \& Freitas, A. (2019). Selection and application of graphical and numerical statistical tools by prospective primary school teachers. Acta Scientiae, 21(6), 82-97.

Fernandes, J. A., Batanero, C., \& Gea, M. M. (2019). Escolha e aplicação de métodos estatísticos por futuros professores dos primeiros anos. In J. M. Contreras, M. M. Gea, M. M. López-Martín, \& E. Molina- Portillo (Ed.), Actas del Tercer Congreso Internacional Virtual de Educación Estadística. Granada: Universidade de Granada.

Fernandes, J. A., Gonçalves, G., \& Barros, P. M. (2021). Uso de tabelas de frequências por futuros professores na realização de trabalhos de projeto. Uniciencia, 35(1).

Fernandes, J. A., Morais, P. C., \& Lacaz, T. V. S. (2011). Representação de dados através de gráficos estatísticos por alunos do $9^{\circ}$ ano de escolaridade. Anais da XIII Conferência Interamericana de Educação Matemática, Recife, Brasil, 2630 junho de 2011.

Friel, S., Curcio, F., \& Bright, G. (2001). Making sense of graphs: critical factors influencing comprehension and instructional implications. Journal for Research in Mathematics Education, 32(2), 124-158.

Godino, J. D., Arteaga, P., Estepa, A., \& Rivas, H. (2013). In J. M. (2013). Contreras, G. R. Cañadas, M. M. Gea, \& P. Arteaga (Eds.), Actas de las Jornadas Virtuales en Didáctica de la Estadística, Probabilidad y Combinatoria (pp. 173-180). Granada, Departamento de Didáctica de la Matemática de la Universidad de Granada.

Gonçalves, G., Fernandes, J. A., \& Gonçalves, J. J. (2020). 
Learning Hypothesis Testing Through a Project Work Methodology. In F. Soares, A. Lopes, K. Brown, \& A. Uukkivi (Eds.), Developing Technology Mediation in Learning Environments (pp. 221-238). Hershey, PA: IGI Global.

Ministério da Educação e Ciência. (2013). Programa de matemática para o ensino básico. Lisboa: Autor.

Morais, P. C., \& Fernandes, J. A. (2011). Realização de duas tarefas sobre construção, leitura e interpretação de gráficos estatísticos por alunos do $9^{\circ}$ ano. In Actas do XXII Seminário de Investigação em Educação Matemática (XXII SIEM). Lisboa: Associação de Professores de Matemática.
Rumsey, D. J. (2002). Statistical literacy as a goal for introductory statistics courses. Journal of Statistics Education, 10(3).

Silva, A. A. (2006). Gráficos e mapas: representação de informação estatística. Lisboa: LIDEL.

Toledo, G. L., \& Ovalle, I. I. (1985). Estatística básica. São Paulo: Atlas.

Tukey, J. W. (1977). Exploratory data analysis. Reading: Addison-Wesley.

Wild, C., \& Pfannkuch, M. (1999). Statistical thinking in empirical enquiry. International Statistical Review, 67(3), 223-248. 